\title{
INEQUALITIES FOR SUPERADDITIVE FUNCTIONALS WITH APPLICATIONS
}

\author{
SEVER S. DRAGOMIR
}

(Received 5 July 2007)

\begin{abstract}
Some inequalities for superadditive functionals defined on convex cones in linear spaces are given, with applications for various mappings associated with the Jensen, Hölder, Minkowski and Schwarz inequalities.
\end{abstract}

2000 Mathematics subject classification: 26D15, 26D10, 46C05, 46B05.

Keywords and phrases: superadditive functionals, convex cones, Jensen's inequality, Hölder's inequality, Minkowski's inequality, Schwarz' inequality.

\section{Introduction}

Let $X$ be a linear space. A subset $C \subseteq X$ is called a convex cone in $X$ provided the following conditions hold:

(i) $x, y \in C$ imply $x+y \in C$;

(ii) $x \in C, \alpha \geq 0$ imply $\alpha x \in C$.

A functional $v: C \rightarrow \mathbb{R}$ is called superadditive on $C$ if

(iii) $v(x+y) \geq v(x)+v(y)$ for any $x, y \in C$,

and nonnegative on $C$ if

(iv) $v(x) \geq 0$ for each $x \in C$.

The functional $v$ is $s$-positive homogeneous on $C$, for a given $s>0$, if

(v) $v(\alpha x)=\alpha^{s} v(x)$ for any $\alpha \geq 0$ and $x \in C$.

The main aim of the present paper is to provide some fundamental inequalities for the values $v(x)$ and $v(y)$ of a superadditive functional $v$ defined on a convex cone $C$ provided that there exist constants $M \geq m>0$ for which $M y-x$ and $x-m y$ remain in $C$. Natural applications in refining some fundamental inequalities such as the Jensen, Hölder, Minkowski and Schwarz inequalities are also provided.

(c) 2008 Australian Mathematical Society 0004-9727/08 \$A2.00+0.00 


\section{The results}

The following fundamental result holds.

THEOREM 1. Let $x, y \in C$, and let $v: C \rightarrow \mathbb{R}$ be a nonnegative, superadditive and s-positive homogeneous functional on $C$. If $M \geq m \geq 0$ are such that $x-m y$ and $M y-x \in C$, then

$$
M^{s} v(y) \geq v(x) \geq m^{s} v(y) .
$$

PROOF. We have successively

$$
\begin{aligned}
v(x) & =v(x-m y+m y) \geq v(x-m y)+v(m y) \\
& =m^{s} v(y)+v(x-m y) \geq m^{s} v(y),
\end{aligned}
$$

giving the second inequality in (1).

For $M=0,(1)$ is obviously true. Suppose that $M>0$. We have successively

$$
\begin{aligned}
v(y) & =v\left(\frac{1}{M} \cdot M y\right)=\frac{1}{M^{s}} v(M y-x+x) \\
& \geq \frac{1}{M^{s}}[v(M y-x)+v(x)] \geq \frac{1}{M^{s}} v(x),
\end{aligned}
$$

giving the first inequality in (1).

Now, let $\ell: C \rightarrow \mathbb{R}$ be an additive and strictly positive functional on $C$ that is also positive homogeneous on $C$, that is,

(vi) $\ell(\alpha x)=\alpha \ell(x)$ for any $\alpha>0$ and $x \in C$.

We have the following result concerning other bounds for a composite functional.

THEOREM 2. Let $x, y \in C$, let $v$ be strictly positive, superadditive and positive homogeneous on $C$, and let $\ell$ be an additive, strictly positive and positive homogeneous functional on $C$. If $M \geq m>0$ are such that $x-m y$ and $M y-x \in C$, then

$$
\left[\frac{v(y)}{\ell(y)}\right]^{M \ell(y)} \geq\left[\frac{v(x)}{\ell(x)}\right]^{\ell(x)} \geq\left[\frac{v(y)}{\ell(y)}\right]^{m \ell(y)} .
$$

Proof. Consider the new functional $\mu: C \rightarrow \mathbb{R}$ defined by

$$
\mu(x):=\ell(x) \ln \left[\frac{v(x)}{\ell(x)}\right] .
$$

Observe that, for $\alpha>0$ and $x \in C$,

$$
\mu(\alpha x)=\ell(\alpha x) \ln \left[\frac{v(\alpha x)}{\ell(\alpha x)}\right]=\alpha \ell(x) \ln \left[\frac{v(x)}{\ell(x)}\right]=\alpha \mu(x),
$$

showing that $\mu$ is positive homogeneous on $C$. 
Using the arithmetic mean-geometric mean inequality,

$$
\frac{\alpha a+\beta b}{\alpha+\beta} \geq a^{(\alpha /(\alpha+\beta))} \cdot b^{(\beta /(\alpha+\beta))},
$$

when $a, b>0, \alpha, \beta \geq 0$ with $\alpha+\beta>0$,

$$
\begin{aligned}
\mu(x+y) & =\ell(x+y) \ln \left[\frac{v(x+y)}{\ell(x+y)}\right] \geq \ell(x+y) \ln \left[\frac{v(x)+v(y)}{\ell(x)+\ell(y)}\right] \\
& =\ell(x+y) \ln \left[\frac{\ell(x) \cdot(v(x) / \ell(x))+\ell(y) \cdot(v(y) / \ell(y))}{\ell(x)+\ell(y)}\right] \\
& \geq[\ell(x)+\ell(y)] \ln \left[\left(\frac{v(x)}{\ell(x)}\right)^{\ell(x) /(\ell(x)+\ell(y))} \cdot\left(\frac{v(y)}{\ell(y)}\right)^{\ell(y) /(\ell(x)+\ell(y))}\right] \\
& =[\ell(x)+\ell(y)]\left\{\frac{\ell(x)}{\ell(x)+\ell(y)} \cdot \ln \left[\frac{v(x)}{\ell(x)}\right]+\frac{\ell(y)}{\ell(x)+\ell(y)} \ln \left[\frac{v(y)}{\ell(y)}\right]\right\} \\
& =\mu(x)+\mu(y),
\end{aligned}
$$

showing that $\mu$ is superadditive on $C$.

Now, if we apply Theorem 1 for $s=1$ and $\mu$, we get

$$
M \ell(y) \ln \left[\frac{v(y)}{\ell(y)}\right] \geq \ell(x) \ln \left[\frac{v(x)}{\ell(x)}\right] \geq m \ell(y) \ln \left[\frac{v(y)}{\ell(y)}\right],
$$

which is clearly equivalent to (2).

\section{Applications for Jensen's inequality}

Let $K$ be a convex subset of the real linear space $X$ and let $f: K \rightarrow \mathbb{R}$ be a convex mapping. Here we consider the following well-known form of Jensen's discrete inequality:

$$
f\left(\frac{1}{P_{I}} \sum_{i \in I} p_{i} x_{i}\right) \leq \frac{1}{P_{I}} \sum_{i \in I} p_{i} f\left(x_{i}\right),
$$

where $I$ denotes a finite subset of the set $\mathbb{N}$ of natural numbers, $x_{i} \in K, p_{i} \geq 0$ for $i \in I$ and $P_{I}:=\sum_{i \in I} p_{i}>0$.

Let us fix $I \in \mathcal{P}_{\mathrm{f}}(\mathbb{N})$ (the class of finite parts of $\mathbb{N}$ ) and $x_{i} \in K(i \in I)$. Now consider the functional $J: S_{+}(I) \rightarrow \mathbb{R}$ given by

$$
J_{I}(p):=\sum_{i \in I} p_{i} f\left(x_{i}\right)-P_{I} f\left(\frac{1}{P_{I}} \sum_{i \in I} p_{i} x_{i}\right) \geq 0,
$$

where $S_{+}(I):=\left\{p=\left(p_{i}\right)_{i \in I} \mid p_{i} \geq 0, i \in I\right.$ and $\left.P_{I}>0\right\}$ and $f$ is convex on $K$.

We observe that $S_{+}(I)$ is a cone and the functional $J_{I}$ is nonnegative, superadditive [3] and positive homogeneous on $S_{+}(I)$.

Using Theorem 1 we can state the following proposition. 
Proposition 3. If $p, q \in S_{+}(I)$ and $M \geq m \geq 0$ are such that $M p \geq q \geq m p$, then

$$
\begin{aligned}
& M\left[\sum_{i \in I} p_{i} f\left(x_{i}\right)-P_{I} f\left(\frac{1}{P_{I}} \sum_{i \in I} p_{i} x_{i}\right)\right] \\
& \quad \geq \sum_{i \in I} q_{i} f\left(x_{i}\right)-Q_{I} f\left(\frac{1}{Q_{I}} \sum_{i \in I} q_{i} x_{i}\right) \\
& \quad \geq m\left[\sum_{i \in I} p_{i} f\left(x_{i}\right)-P_{I} f\left(\frac{1}{P_{I}} \sum_{i \in I} p_{i} x_{i}\right)\right] \quad(\geq 0) .
\end{aligned}
$$

Now, on choosing $v(p):=J_{I}(p)$ and $\ell(p):=P_{I}$ and applying Theorem 2, we can state the following result as well.

Proposition 4. With the assumptions of Proposition 3,

$$
\begin{aligned}
{\left[\frac{1}{P_{I}} \sum_{i \in I} p_{i} f\left(x_{i}\right)-f\left(\frac{1}{P_{I}} \sum_{i \in I} p_{i} x_{i}\right)\right]^{M P_{I}} } \\
\quad \geq\left[\frac{1}{Q_{I}} \sum_{i \in I} q_{i} f\left(x_{i}\right)-f\left(\frac{1}{Q_{I}} \sum_{i \in I} q_{i} x_{i}\right)\right]^{Q_{I}} \\
\quad \geq\left[\frac{1}{P_{I}} \sum_{i \in I} p_{i} f\left(x_{i}\right)-f\left(\frac{1}{P_{I}} \sum_{i \in I} p_{i} x_{i}\right)\right]^{m P_{I}} .
\end{aligned}
$$

The above results can be used to obtain various inequalities generated by the appropriate choices of the convex function $f$.

(1) If $f: X \rightarrow \mathbb{R}, f(x)=\|x\|^{r}, r \geq 1$, where $(X,\|\cdot\|)$ is a normed linear space, then

$$
\begin{aligned}
& M\left[\sum_{i \in I} p_{i}\left\|x_{i}\right\|^{r}-P_{I}^{1-r}\left\|\sum_{i \in I} p_{i} x_{i}\right\|^{r}\right] \\
& \quad \geq \sum_{i \in I} q_{i}\left\|x_{i}\right\|^{r}-Q_{I}^{1-r}\left\|\sum_{i \in I} q_{i} x_{i}\right\|^{r} \\
& \quad \geq m\left[\sum_{i \in I} p_{i}\left\|x_{i}\right\|^{r}-P_{I}^{1-r}\left\|\sum_{i \in I} p_{i} x_{i}\right\|^{r}\right],
\end{aligned}
$$


and

$$
\begin{aligned}
\frac{Q_{I}^{r Q_{I}}}{P_{I}^{r M P_{I}}} & {\left[P_{I}^{r-1} \sum_{i \in I} p_{i}\left\|x_{i}\right\|^{r}-\left\|\sum_{i \in I} p_{i} x_{i}\right\|^{r}\right]^{M P_{I}} } \\
\geq & {\left[Q_{I}^{r-1} \sum_{i \in I} q_{i}\left\|x_{i}\right\|^{r}-\left\|\sum_{i \in I} q_{i} x_{i}\right\|^{r}\right]^{Q_{I}} } \\
\geq & \frac{Q_{I}^{r Q_{I}}}{P_{I}^{r m P_{I}}}\left[P_{I}^{r-1} \sum_{i \in I} p_{i}\left\|x_{i}\right\|^{r}-\left\|\sum_{i \in I} p_{i} x_{i}\right\|^{r}\right]^{m P_{I}},
\end{aligned}
$$

for $I \in \mathcal{P}_{\mathrm{f}}(\mathbb{N})$ and $p, q \in S_{+}(I)$ with $M p \geq q \geq m p$ and $M \geq m>0$ and for any vectors $x_{i} \in X, i \in I$.

(2) For $x_{i}>0$ and $p_{i} \geq 0(i \in \mathbb{N})$ such that $P_{I}>0$, let

$$
A(I, p, x):=\frac{1}{P_{I}} \sum_{i \in I} p_{i} x_{i}, \quad G(I, p, x):=\left(\prod_{i \in I}\left(x_{i}\right)^{p_{i}}\right)^{1 / P_{I}}
$$

denote the weighted arithmetic and geometric means respectively.

Applying the above two propositions for the convex function $f(x)=-\ln x$, $x \in(0, \infty)$, we can state the following inequalities:

$$
\left[\frac{A(I, p, x)}{G(I, p, x)}\right]^{M P_{I}} \geq\left[\frac{A(I, p, x)}{G(I, p, x)}\right]^{Q_{I}} \geq\left[\frac{A(I, p, x)}{G(I, p, x)}\right]^{m P_{I}}
$$

and

$$
\left\{\ln \left[\frac{A(I, p, x)}{G(I, p, x)}\right]\right\}^{M P_{I}} \geq\left\{\ln \left[\frac{A(I, p, x)}{G(I, p, x)}\right]\right\}^{Q_{I}} \geq\left\{\ln \left[\frac{A(I, p, x)}{G(I, p, x)}\right]\right\}^{m P_{I}},
$$

for $I \in \mathcal{P}_{\mathrm{f}}(\mathbb{N})$ and $p, q \in S_{+}(I)$ with $M p \geq q \geq m p$ and $M \geq m>0$ and for any $x_{i}>0, i \in I$.

\section{Applications for Hölder's inequality in normed spaces}

Let $(X,\|\cdot\|)$ be a normed space and $I \in \mathcal{P}_{\mathrm{f}}(\mathbb{N})$. We define

$$
\begin{aligned}
E(I) & :=\left\{x=\left(x_{j}\right)_{j \in I} \mid x_{j} \in X, j \in I\right\}, \\
\mathbb{K}(I) & :=\left\{\lambda=\left(\lambda_{j}\right)_{j \in I} \mid \lambda_{j} \in \mathbb{K}, j \in I\right\} .
\end{aligned}
$$

We consider for $\alpha, \beta>1,(1 / \alpha)+(1 / \beta)=1$ the functional

$$
H_{I}(p, \lambda, x ; \alpha, \beta):=\left(\sum_{j \in I} p_{j}\left|\lambda_{j}\right|^{\alpha}\right)^{1 / \alpha}\left(\sum_{j \in I} p_{j}\left\|x_{j}\right\|^{\beta}\right)^{1 / \beta}-\left\|\sum_{j \in I} p_{j} \lambda_{j} x_{j}\right\| .
$$


Lemma 5. For any $p, q \in S_{+}(I)$,

$$
H_{I}(p+q, \lambda, x ; \alpha, \beta) \geq H_{I}(p, \lambda, x ; \alpha, \beta)+H_{I}(q, \lambda, x ; \alpha, \beta),
$$

where $x \in E(I), \lambda \in \mathbb{K}(I)$ and $\alpha, \beta>1$ with $(1 / \alpha)+(1 / \beta)=1$.

PROOF. Using the elementary inequality

$$
\left(a^{\alpha}+b^{\alpha}\right)^{1 / \alpha}\left(c^{\beta}+d^{\beta}\right)^{1 / \beta} \geq a c+b d,
$$

with $\alpha, \beta>1,(1 / \alpha)+(1 / \beta)=1$ and $a, b, c, d \geq 0$, and the triangle inequality,

$$
\begin{aligned}
H_{I}(p+ & q, \lambda, x ; \alpha, \beta) \\
= & \left(\sum_{i \in I} p_{i}\left|\lambda_{i}\right|^{\alpha}+\sum_{i \in I} q_{i}\left|\lambda_{i}\right|^{\alpha}\right)^{1 / \alpha}\left(\sum_{i \in I} p_{i}\left\|x_{i}\right\|^{\beta}+\sum_{i \in I} q_{i}\left\|x_{i}\right\|^{\beta}\right)^{1 / \beta} \\
& -\left\|\sum_{i \in I} p_{i} \lambda_{i} x_{i}+\sum_{i \in I} q_{i} \lambda_{i} x_{i}\right\| \\
= & \left\{\left[\left(\sum_{i \in I} p_{i}\left|\lambda_{i}\right|^{\alpha}\right)^{1 / \alpha}\right]^{\alpha}+\left[\left(\sum_{i \in I} q_{i}\left|\lambda_{i}\right|^{\alpha}\right)^{1 / \alpha}\right]^{\alpha}\right\} \\
& \times\left\{\left[\left(\sum_{i \in I} p_{i}\left\|x_{i}\right\|^{\beta}\right)^{1 / \beta}\right]^{\beta}+\left[\left(\sum_{i \in I} q_{i}\left\|x_{i}\right\|^{\beta}\right)^{1 / \beta}\right]^{\beta}\right\} \\
& -\left\|\sum_{i \in I} p_{i} \lambda_{i} x_{i}+\sum_{i \in I} q_{i} \lambda_{i} x_{i}\right\| \\
\geq & \left(\sum_{i \in I} p_{i}\left|\lambda_{i}\right|^{\alpha}\right)^{1 / \alpha}\left(\sum_{i \in I} p_{i}\left\|x_{i}\right\|^{\beta}\right)^{1 / \beta} \\
& +\left(\sum_{i \in I} q_{i}\left|\lambda_{i}\right|^{\alpha}\right)^{1 / \alpha}\left(\sum_{i \in I} q_{i}\left\|x_{i}\right\|^{\beta}\right)^{1 / \beta}-\left\|\sum_{i \in I} p_{i} \lambda_{i} x_{i}\right\|-\left\|\sum_{i \in I} q_{i} \lambda_{i} x_{i}\right\| \\
= & H_{I}(p, \lambda, x ; \alpha, \beta)+H_{I}(q, \lambda, x ; \alpha, \beta),
\end{aligned}
$$

and the superadditivity of $H$ is proved.

REMARK 1 . The same result can be stated if $(B,\|\cdot\|)$ is a normed algebra and the functional $H$ is defined by

$$
H_{I}(p, \lambda, x ; \alpha, \beta):=\left(\sum_{i \in I} p_{i}\left\|x_{i}\right\|^{\alpha}\right)^{1 / \alpha}\left(\sum_{i \in I} p_{i}\left\|y_{i}\right\|^{\beta}\right)^{1 / \beta}-\left\|\sum_{i \in I} p_{i} x_{i} y_{i}\right\|,
$$

where $\quad x=\left(x_{i}\right)_{i \in I}, y=\left(y_{i}\right)_{i \in I} \subset B, \quad p \in S_{+}(I) \quad$ and $\quad \alpha, \beta>1 \quad$ with $(1 / \alpha)+(1 / \beta)=1$. 
Since, obviously, $H(\cdot, \lambda, x ; \alpha, \beta)$ is positive homogeneous, on using Theorems 1 and 2 , we can state the following propositions.

Proposition 6. If $p, q \in S_{+}(I)$ and $M \geq m \geq 0$ with $M p \geq q \geq m p$, then

$$
\begin{aligned}
M[ & \left.\left(\sum_{i \in I} p_{i}\left|\lambda_{i}\right|^{\alpha}\right)^{1 / \alpha}\left(\sum_{i \in I} p_{i}\left\|x_{i}\right\|^{\beta}\right)^{1 / \beta}-\left\|\sum_{i \in I} p_{i} \lambda_{i} x_{i}\right\|\right] \\
& \geq\left(\sum_{i \in I} q_{i}\left|\lambda_{i}\right|^{\alpha}\right)^{1 / \alpha}\left(\sum_{i \in I} q_{i}\left\|x_{i}\right\|^{\beta}\right)^{1 / \beta}-\left\|\sum_{i \in I} q_{i} \lambda_{i} x_{i}\right\| \\
& \geq m\left[\left(\sum_{i \in I} p_{i}\left|\lambda_{i}\right|^{\alpha}\right)^{1 / \alpha}\left(\sum_{i \in I} p_{i}\left\|x_{i}\right\|^{\beta}\right)^{1 / \beta}-\left\|\sum_{i \in I} p_{i} \lambda_{i} x_{i}\right\|\right] \quad(\geq 0) .
\end{aligned}
$$

Now, for $\ell(p):=P_{I}$ and $v(p)=H_{I}(p, \lambda, x ; \alpha, \beta)$, on applying Theorem 2 , we have the following.

Proposition 7. With the assumptions in Proposition 6,

$$
\begin{aligned}
& {\left[\left(\frac{1}{P_{I}} \sum_{i \in I} p_{i}\left|\lambda_{i}\right|^{\alpha}\right)^{1 / \alpha}\left(\frac{1}{P_{I}} \sum_{i \in I} p_{i}\left\|x_{i}\right\|^{\beta}\right)^{1 / \beta}-\left\|\frac{1}{P_{I}} \sum_{i \in I} p_{i} \lambda_{i} x_{i}\right\|\right]^{M P_{I}}} \\
& \quad \geq\left[\left(\frac{1}{Q_{I}} \sum_{i \in I} q_{i}\left|\lambda_{i}\right|^{\alpha}\right)^{1 / \alpha}\left(\frac{1}{Q_{I}} \sum_{i \in I} q_{i}\left\|x_{i}\right\|^{\beta}\right)^{1 / \beta}-\left\|\frac{1}{Q_{I}} \sum_{i \in I} q_{i} \lambda_{i} x_{i}\right\|\right]^{Q_{I}} \\
& \quad \geq\left[\left(\frac{1}{P_{I}} \sum_{i \in I} p_{i}\left|\lambda_{i}\right|^{\alpha}\right)^{1 / \alpha}\left(\frac{1}{P_{I}} \sum_{i \in I} p_{i}\left\|x_{i}\right\|^{\beta}\right)^{1 / \beta}-\left\|\frac{1}{P_{I}} \sum_{i \in I} p_{i} \lambda_{i} x_{i}\right\|\right]^{m P_{I}},
\end{aligned}
$$

for $x \in E(I), \lambda \in \mathbb{K}(I)$ and $\alpha, \beta>1,(1 / \alpha)+(1 / \beta)=1$.

Similar results may be stated for normed algebras. However, the details are omitted.

\section{Applications for Minkowski's inequality}

Let $(X,\|\cdot\|)$ be a normed space and $I \in \mathcal{P}_{\mathrm{f}}(\mathbb{N})$. We define the functional

$$
M_{I}(p, x, y ; \alpha)=\left[\left(\sum_{i \in I} p_{i}\left\|x_{i}\right\|^{\alpha}\right)^{1 / \alpha}+\left(\sum_{i \in I} p_{i}\left\|y_{i}\right\|^{\alpha}\right)^{1 / \alpha}\right]^{\alpha}-\sum_{i \in I} p_{i}\left\|x_{i}+y_{i}\right\|^{\alpha},
$$

where $p \in S_{+}(I), \alpha \geq 1$ and $x, y \in E(I)$.

LEMMA 8. For any $p, q \in S_{+}(I)$,

$$
M_{I}(p+q, x, y ; \alpha) \geq M_{I}(p, x, y ; \alpha)+M_{I}(q, x, y ; \alpha),
$$

where $x, y \in E(I)$ and $\alpha \geq 1$. 
Proof. Using the elementary inequality

$$
\left(a^{\alpha}+b^{\alpha}\right)^{1 / \alpha}+\left(c^{\alpha}+d^{\alpha}\right)^{1 / \alpha} \geq\left[(a+c)^{\alpha}+(b+d)^{\alpha}\right]^{1 / \alpha},
$$

for $a, b, c, d \geq 0$ and $\alpha \geq 1$,

$$
\begin{aligned}
M_{I}(p+q, x, y ; \alpha)= & {\left[\left\{\left[\left(\sum_{i \in I} p_{i}\left\|x_{i}\right\|^{\alpha}\right)^{1 / \alpha}\right]^{\alpha}+\left[\left(\sum_{i \in I} q_{i}\left\|x_{i}\right\|^{\alpha}\right)^{1 / \alpha}\right]^{\alpha}\right\}^{1 / \alpha}\right.} \\
& \left.+\left\{\left[\left(\sum_{i \in I} p_{i}\left\|y_{i}\right\|^{\alpha}\right)^{1 / \alpha}\right]^{\alpha}+\left[\left(\sum_{i \in I} q_{i}\left\|y_{i}\right\|^{\alpha}\right)^{1 / \alpha}\right]^{\alpha}\right\}^{1 / \alpha}\right]^{\alpha} \\
& -\sum_{i \in I} p_{i}\left\|x_{i}+y_{i}\right\|^{\alpha}-\sum_{i \in I} q_{i}\left\|x_{i}+y_{i}\right\|^{\alpha} \\
\geq & {\left[\left(\sum_{i \in I} p_{i}\left\|x_{i}\right\|^{\alpha}\right)^{1 / \alpha}+\left(\sum_{i \in I} p_{i}\left\|y_{i}\right\|^{\alpha}\right)^{1 / \alpha}\right]^{\alpha} } \\
& +\left[\left(\sum_{i \in I} q_{i}\left\|x_{i}\right\|^{\alpha}\right)^{1 / \alpha}+\left(\sum_{i \in I} q_{i}\left\|y_{i}\right\|^{\alpha}\right)^{1 / \alpha}\right]^{\alpha} \\
& -\sum_{i \in I} p_{i}\left\|x_{i}+y_{i}\right\|^{\alpha}-\sum_{i \in I} q_{i}\left\|x_{i}+y_{i}\right\|^{\alpha} \\
= & M_{I}(p, x, y ; \alpha)+M_{I}(q, x, y ; \alpha),
\end{aligned}
$$

which proves the superadditivity property of the functional $M$.

Since the functional $M_{I}(\cdot, x, y ; \alpha)$ is positive homogeneous on $S_{+}(I)$, on using Theorem 1 , we can state the following proposition.

Proposition 9. If $p, q \in S_{+}(I)$ and $M \geq m \geq 0$ with $M p \geq q \geq m p$, then

$$
\begin{aligned}
M\{ & {\left.\left[\left(\sum_{i \in I} p_{i}\left\|x_{i}\right\|^{\alpha}\right)^{1 / \alpha}+\left(\sum_{i \in I} p_{i}\left\|y_{i}\right\|^{\alpha}\right)^{1 / \alpha}\right]^{\alpha}-\sum_{i \in I} p_{i}\left\|x_{i}+y_{i}\right\|^{\alpha}\right\} } \\
& \geq\left[\left(\sum_{i \in I} q_{i}\left\|x_{i}\right\|^{\alpha}\right)^{1 / \alpha}+\left(\sum_{i \in I} q_{i}\left\|y_{i}\right\|^{\alpha}\right)^{1 / \alpha}\right]^{\alpha}-\sum_{i \in I} q_{i}\left\|x_{i}+y_{i}\right\|^{\alpha} \\
& \geq m\left\{\left[\left(\sum_{i \in I} p_{i}\left\|x_{i}\right\|^{\alpha}\right)^{1 / \alpha}+\left(\sum_{i \in I} p_{i}\left\|y_{i}\right\|^{\alpha}\right)^{1 / \alpha}\right]^{\alpha}-\sum_{i \in I} p_{i}\left\|x_{i}+y_{i}\right\|^{\alpha}\right\} .
\end{aligned}
$$

Now, since $\ell(p)=P_{I}$ is additive and positive homogeneous on $S_{+}(I)$, on using Theorem 2 we can state the following result as well. 
Proposition 10. With the assumptions in Proposition 9,

$$
\begin{aligned}
\{[ & \left.\left.\left.\frac{1}{P_{I}} \sum_{i \in I} p_{i}\left\|x_{i}\right\|^{\alpha}\right)^{1 / \alpha}+\left(\frac{1}{P_{I}} \sum_{i \in I} p_{i}\left\|y_{i}\right\|^{\alpha}\right)^{1 / \alpha}\right]^{\alpha}-\frac{1}{P_{I}} \sum_{i \in I} p_{i}\left\|x_{i}+y_{i}\right\|^{\alpha}\right\}^{M P_{I}} \\
\geq & \left\{\left(\frac{1}{Q_{I}} \sum_{i \in I} q_{i}\left\|x_{i}\right\|^{\alpha}\right)^{1 / \alpha}+\left(\frac{1}{Q_{I}} \sum_{i \in I} q_{i}\left\|y_{i}\right\|^{\alpha}\right)^{1 / \alpha}\right]^{\alpha} \\
& \left.-\frac{1}{Q_{I}} \sum_{i \in I} q_{i}\left\|x_{i}+y_{i}\right\|^{\alpha}\right\}^{Q_{I}} \\
\geq & \left\{\left[\left(\frac{1}{P_{I}} \sum_{i \in I} p_{i}\left\|x_{i}\right\|^{\alpha}\right)^{1 / \alpha}+\left(\frac{1}{P_{I}} \sum_{i \in I} p_{i}\left\|y_{i}\right\|^{\alpha}\right)^{1 / \alpha}\right]^{\alpha}\right. \\
& \left.-\frac{1}{P_{I}} \sum_{i \in I} p_{i}\left\|x_{i}+y_{i}\right\|^{\alpha}\right\}^{m P_{I}} .
\end{aligned}
$$

\section{Applications for the Schwarz inequality}

Let $X$ be a linear space over the real or complex number field $\mathbb{K}$ and let us denote by $\mathcal{H}(X)$ the class of all positive-semi-definite Hermitian forms on $X$, or, for simplicity, nonnegative forms on $X$ : that is, the mapping $\langle\cdot, \cdot\rangle: X \times X \rightarrow \mathbb{K}$ belongs to $\mathcal{H}(X)$ if it satisfies the conditions:

(i) $\langle x, x\rangle \geq 0$ for all $x \in X$;

(ii) $\langle\alpha x+\beta y, z\rangle=\alpha\langle x, z\rangle+\beta\langle y, z\rangle$ for all $x, y \in X$ and $\alpha, \beta \in \mathbb{K}$;

(iii) $\langle y, x\rangle=\overline{\langle x, y\rangle}$ for all $x, y \in X$.

If $\langle\cdot, \cdot\rangle \in \mathcal{H}(X)$, then the functional $\|\cdot\|=\langle\cdot, \cdot\rangle^{1 / 2}$ is a semi-norm on $X$ and the following version of Schwarz' inequality holds:

$$
\|x\|\|y\| \geq|\langle x, y\rangle|
$$

for each $x, y \in H$.

Now, let us observe that $\mathcal{H}(X)$ is a convex cone in the linear space of all mappings defined on $X^{2}$ with values in $\mathbb{K}$. Also, we can introduce on $\mathcal{H}(X)$ the following binary relation [2]

$$
\langle\cdot, \cdot\rangle_{2} \geq\langle\cdot, \cdot\rangle_{1} \quad \text { if and only if } \quad\|x\|_{2} \geq\|x\|_{1} \quad \text { for any } x \in H .
$$

This is an order relation on $\mathcal{H}(X)$.

Consider the functional [2]

$$
\sigma: \mathcal{H}(X) \times X^{2} \rightarrow \mathbb{R}_{+}, \quad \sigma(\langle\cdot, \cdot\rangle ; x, y):=\|x\|\|y\|-|\langle x, y\rangle|,
$$

which is closely related to the second version of the Schwarz inequality in (13). 
LEMMA 11 (Dragomir-Mond [2]). The functional $\sigma(\cdot ; x, y)$ is nonnegative, superadditive and positive homogeneous on $\mathcal{H}(X)$.

Proposition 12. Let $M \geq m>0$, and let $\langle\cdot, \cdot\rangle_{1},\langle\cdot, \cdot\rangle_{2}$ be two nonnegative Hermitian forms on $X$ such that $M\|x\|_{1} \geq\|x\|_{2} \geq m\|x\|_{1}$ for each $x \in X$. Then

$$
\begin{aligned}
M^{2}\left(\|x\|_{1}\|y\|_{1}-\left|\langle x, y\rangle_{1}\right|\right) & \geq\|x\|_{2}\|y\|_{2}-\left|\langle x, y\rangle_{2}\right| \\
& \geq m^{2}\left(\|x\|_{1}\|y\|_{1}-\left|\langle x, y\rangle_{1}\right|\right),
\end{aligned}
$$

for any $x, y \in H$.

PROOF. From the hypothesis, $M^{2}\langle\cdot, \cdot\rangle_{2}-\langle\cdot, \cdot\rangle_{1}$ and $\langle\cdot, \cdot\rangle_{2}-m^{2}\langle\cdot, \cdot\rangle_{1}$ are nonnegative Hermitian forms. Then applying Theorem 1 for the functional

$$
\sigma(\langle\cdot, \cdot\rangle ; x, y):=\|x\|\|y\|-|\langle x, y\rangle|,
$$

for $x, y$ fixed in $X$, we deduce the desired result.

REMARK 2. If we assume that $A: H \rightarrow H$ is a self-adjoint linear operator on the Hilbert space $(H,\langle\cdot, \cdot\rangle)$ satisfying the property that there exist $P \geq p>0$ such that $P I \geq A \geq p I$ in the operation order (that is, $P\|x\|^{2} \geq\langle A x, x\rangle \geq p\|x\|^{2}$ for any $x \in H$ ), then we have the inequality

$$
\begin{aligned}
P(\|x\|\|y\|-|\langle x, y\rangle|) & \geq\langle A x, x\rangle^{1 / 2}\langle A y, y\rangle^{1 / 2}-|\langle A x, y\rangle| \\
& \geq p(\|x\|\|y\|-|\langle x, y\rangle|),
\end{aligned}
$$

for any $x, y \in H$.

For $e \in X, e \neq 0$ we can define the functional

$$
\ell(\langle\cdot, \cdot\rangle ; e):=\|e\|^{2}=\langle e, e\rangle .
$$

For fixed $e \in H$, the functional $\ell(\cdot ; e)$ is additive and positive homogeneous on $\mathcal{H}(X)$.

Using Theorem 2, we can state the following result as well.

Proposition 13. Let $M \geq m>0$, and let $\langle\cdot, \cdot\rangle_{1},\langle\cdot, \cdot\rangle_{2}$ be two inner products on $X$ such that $M\|x\|_{1} \geq\|x\|_{2} \geq m\|x\|_{1}$ for each $x \in H$. Then for any $e \in X, e \neq 0$,

$$
\begin{aligned}
{\left[\frac{\|x\|_{1}\|y\|_{1}-\left|\langle x, y\rangle_{1}\right|}{\|e\|_{1}^{2}}\right]^{M\|e\|_{1}^{2}} } & \geq\left[\frac{\|x\|_{2}\|y\|_{2}-\left|\langle x, y\rangle_{2}\right|}{\|e\|_{2}^{2}}\right]^{\|e\|_{2}^{2}} \\
& \geq\left[\frac{\|x\|_{1}\|y\|_{1}-\left|\langle x, y\rangle_{1}\right|}{\|e\|_{1}^{2}}\right]^{m\|e\|_{1}^{2}} .
\end{aligned}
$$


REMARK 3. Similar results can be stated if one uses the following nonnegative, superadditive and $s$-positive homogeneous functionals on $\mathcal{H}(X)$ (see [1, pp. 8-15]):

$$
\begin{aligned}
\sigma_{\mathrm{r}}(\langle\cdot, \cdot\rangle ; x, y) & :=\|x\|\|y\|-\operatorname{Re}\langle x, y\rangle ; \\
\delta(\langle\cdot, \cdot\rangle ; x, y) & :=\|x\|^{2}\|y\|^{2}-|\langle x, y\rangle|^{2} ; \\
\delta_{\mathrm{r}}(\langle\cdot, \cdot\rangle ; x, y) & :=\|x\|^{2}\|y\|^{2}-(\operatorname{Re}\langle x, y\rangle)^{2} ; \\
\gamma(\langle\cdot, \cdot\rangle ; x, y) & :=\frac{\|x\|^{2}\|y\|^{2}-|\langle x, y\rangle|^{2}}{\|y\|^{2}} ;
\end{aligned}
$$

where in the definition of $\gamma,\langle\cdot, \cdot\rangle$ is an inner product and $y$ is not zero, and

$$
\beta(\langle\cdot, \cdot\rangle ; x, y):=\left(\|x\|^{2}\|y\|^{2}-|\langle x, y\rangle|^{2}\right)^{1 / 2},
$$

for each $x, y \in X$.

The details are left to the interested reader.

\section{References}

[1] S. S. Dragomir, Advances in Inequalities of the Schwarz, Triangle and Heisenberg Type in Inner Product Spaces (Nova Science Publishers, New York, 2007).

[2] S. S. Dragomir and B. Mond, 'On the superadditivity and monotonicity of Schwarz's inequality in inner product spaces', Makedon Akad. Nauk. Umet. Oddel. Mat.-Tehn. Nauk. Prilozi 15(2) (1994), $5-22$.

[3] S. S. Dragomir, J. Pečarić and L. E. Persson, 'Properties of some functionals related to Jensen's inequality', Acta Math. Hungar. 70 (1996), 129-143.

SEVER S. DRAGOMIR, School of Computer Science and Mathematics, Victoria University, PO Box 14428, Melbourne City, 8001, VIC, Australia e-mail: sever.dragomir@vu.edu.au 